\title{
Low-cost in vitro fertilization: current insights
}

This article was published in the following Dove Press journal:

International Journal of Women's Health

2I August 2014

Number of times this article has been viewed

Pek Joo Teoh

Abha Maheshwari

Aberdeen Fertility Centre, Aberdeen Maternity Hospital, University of

Aberdeen, Aberdeen, UK
Correspondence: Pek Joo Teoh Aberdeen Fertility Centre, Aberdeen Maternity Hospital, Foresterhill, Aberdeen AB25 2ZL, UK

Tel +44 I224550567

Email joo.teoh@yahoo.com
Abstract: Despite the development of in vitro fertilization (IVF) more than 30 years ago, the cost of treatment remains high. Furthermore, over the years, more sophisticated technologies and expensive medications have been introduced, making IVF increasingly inaccessible despite the increasing need. Globally, the option to undergo IVF is only available to a privileged few. In recent years, there has been growing interest in exploring strategies to reduce the cost of IVF treatment, which would allow the service to be provided in low-resource settings. In this review, we explore the various ways in which the cost of this treatment can be reduced.

Keywords: IVF, low-cost, accessible, developing world

\section{Introduction}

Since the first test tube baby was born in the year 1978, in vitro fertilization (IVF) treatment has been developed and improved to benefit millions of people who experience childlessness. It is estimated that the number of IVF infants in the world, including intracytoplasmic sperm injection, has now surpassed five million. However, there are still many people who are unable to gain access to this form of treatment.

\section{High treatment cost: a barrier to accessing IVF treatment}

The high cost of IVF treatment is the main barrier that prevents many from benefiting from this technology to help them conceive. Although the cost of IVF treatment is variable among different countries, it is generally an expensive treatment in most parts of the world. The average cost of one fresh IVF cycle (excluding associated frozen embryo transfers) is as high as $£ 3,123$ in the United Kingdom; ; 1,2 in the United States, it is even higher (Table 1). Public funding and the provision of health insurance for IVF treatment differ widely between countries; there is no uniformity in the provision of this treatment even in the developed world. ${ }^{3}$ In Australia, treatment for IVF is publicly funded with no limitations on the number of previous cycles, maternal age, duration of subfertility, body mass index, or smoking status; however, patients are required to make copayments for their treatments. In 2010, the amount of copayment required for a fresh IVF cycle was increased from approximately $\$ 1,500$ to $\$ 2,500$ Australian dollars. As a result, there was a $21 \%-25 \%$ reduction in fresh IVF cycles across all socioeconomic groups. ${ }^{4}$ In contrast to Australia, access to IVF in the United States is only available via private funding. Analysis of data from a prospective cohort in the United States revealed that higher-income and college-educated couples were 
Table I The costs per IVF/ICSI cycle in different countries

\begin{tabular}{|c|c|}
\hline Country & Costs per IVF/ICSI cycle (USD) \\
\hline Australia & 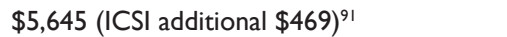 \\
\hline Brazil & $\$ 3,000^{9}$ \\
\hline Canada & $\$ 8,500(\text { ICSI additional } \$ 1,172)^{91}$ \\
\hline Germany & $\$ 4, \mid 48-\$ 4,977 *, 6$ \\
\hline India & $\$ 600-\$ 1,000^{92}$ \\
\hline Japan & $\$ 3,956(\text { ICSI additional } \$ 860)^{91}$ \\
\hline Scandinavian countries & 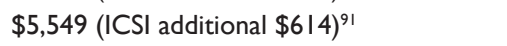 \\
\hline \multicolumn{2}{|l|}{ South Africa } \\
\hline IVF & $\$ 4,500 \pm \$ 796$ (mean \pm standard deviation) $)^{*, 93}$ \\
\hline ICSI & $\$ 4,565 \pm \$ 864$ (mean \pm standard deviation) $)^{*, 93}$ \\
\hline United Kingdom & $\$ 5,244^{*, 1,2}$ \\
\hline United States & $\$ 12,513(\mathrm{ICSI} \text { additional } \$ 1,626)^{91}$ \\
\hline
\end{tabular}

Notes: *Currency conversion based on the exchange rates on April 28, 2014 (http://www.xe.com). Rounded to the nearest dollar.

Abbreviations: ICSI intra-cytoplasmic sperm injection; IVF, in vitro fertilization.

much more likely to have multiple cycles of IVF treatment. ${ }^{5}$ Many other countries, for example the United Kingdom, incur strict restrictions and limitations on the provision of IVF treatment via public funding. In addition, the number of cycles funded is often limited for each person due to the cost involved. Germany, in some way similar to Australia, provides partial reimbursement to meet some of the cost of IVF treatment. Even with the provision of funding of up to $50 \%$, three quarters of couples in Germany declined to proceed to IVF treatment; these patients cited cost of treatment as one of the major reasons that stopped them from undergoing IVF. 3,6

\section{IVF in developing countries}

The availability of IVF in developing countries is primarily limited to a few privileged individuals who can afford to self-fund the cost involved. Publicly funded treatments are scarcely available; in many countries, they are nonexistent. According to a survey conducted in Kenya, more than a quarter of patients attending consultations are affected by subfertility. ${ }^{7}$ The survey revealed that in Kenya only three units provide IVF, and only a minority of patients were able to gain access to IVF treatment via private funding. In Brazil, $76 \%$ of the states have no fully subsidized treatment for subfertility. ${ }^{8}$ The charges for the treatment are unaffordable for the majority of the low-income Brazilian population, and the waiting time for access to assisted reproductive treatment can be as long as 6 years. ${ }^{9}$

\section{Subfertility as a global health issue}

Subfertility has been overlooked as a global health issue, despite affecting one in seven couples. In developing countries, resources are often channeled to tackle other major health problems like malnutrition and infectious diseases. Being a non-life-threatening condition, subfertility is not on the priority lists of most policy makers. ${ }^{8}$ Many countries are also facing the problem of high population growth rates; currently, there is more emphasis on family planning than on the issue of subfertility in these countries. For most international nonprofit organizations, the implementation of appropriate treatment for subfertility is not part of their main objectives. ${ }^{10}$

In many cultures in the developing world, child bearing is considered the central purpose in life. Subfertile couples not only have to bear the sorrow of childlessness, but they are also subjected to social stigma, become isolated from family and community, and have their social and cultural rights stripped. ${ }^{3}$ Women tend to be blamed for the subfertility; the situation can result in domestic violence and polygamy.,10 Research has shown that psychological distress and physical abuse is significantly more common in subfertile women than in their fertile counterparts. ${ }^{11}$ As a result of pregnancyrelated or delivery-related infections, unsafe abortions, and the high incidence of sexually transmitted diseases, bilateral tubal blockage is the most common cause of subfertility in developing countries; IVF treatment is the most effective intervention for this condition. ${ }^{10,12}$ However, in resource-poor settings, this high-priced treatment is typically unavailable, and even individuals who can afford the treatment may have to travel overseas to receive this medical therapy.

\section{Global attempts at lowering the costs of IVF}

The affordability of IVF treatment for individuals of lower socioeconomic background has only very recently received some attention. For more than 10 years, subfertility in developing countries has been highlighted as an important public health issue. However, over the years, there has been little progress in subfertility prevention and treatment in these countries, and the allocation of resources into subfertilityrelated reproductive health programs has been very limited. In 2001, the World Health Organization organized a meeting entitled "Medical, Ethical and Social Aspects of Assisted Reproduction". Recommendations stated in the meeting include the following: 1) infertility should be recognized as a public health issue worldwide, including in developing countries; 2) policy makers and health staff should give attention to infertility and the needs of infertile patients; 3 ) infertility management should be integrated into national reproductive health education programs and services; and 4) assisted reproduction treatment should be complementary 
to other ethically acceptable social and cultural solutions to infertility. ${ }^{12}$ To further increase the awareness among subfertility specialists of the need for accessible subfertility care in developing countries, the European Society of Human Reproduction and Embryology founded a special task force on "Developing Countries and Infertility" in 2006. ${ }^{12}$ Shortly after, The Walking $\operatorname{Egg}^{13}$ was founded. The Walking Egg is a nonprofit organization that aims to establish subfertility services in developing countries. In cooperation with the European Society of Human Reproduction and Embryology and the World Health Organization, The Walking Egg started a global project with the goal to increase diagnostic and therapeutic options, including IVF, for childless couples in resource-poor countries. ${ }^{12}$ This project was conceived in an important meeting organized by The Walking Egg in Arusha, Tanzania in December 2007. One of the most important strategies to improve accessibility of fertility treatment is lowering the cost of IVF, representing an effective method to improve the affordability of this therapeutic option for people with lower socioeconomic status in both the developing world and countries with advanced economies.

\section{Expenditures in IVF treatment}

Not including the fee for service, the high price tag for IVF treatment is largely due to expenditures for investigations, medications, and laboratorial equipment. The cost of treating the adverse effects from IVF can also incur additional strain on healthcare resources, hence limiting public funding for IVF. Lately, more economical approaches in providing IVF treatment have been discussed and researched. Cheaper alternatives to the expensive protocols used frequently in various IVF centers have been tested to assess their safety and effectiveness (Table 2).

\section{Is IVF really needed?}

Recently, concerns have arisen as to whether IVF is being performed unnecessarily, especially in the group of patients with a short duration of unexplained subfertility. ${ }^{14}$ Unexplained

Table 2 The expenditure and strategies to reduce cost in IVF

\begin{tabular}{ll}
\hline Expenditure in IVF & Strategies to reduce cost \\
\hline Offering unnecessary IVF & Careful patient selection \\
Investigations & Simplifying investigative methods \\
Medications & Reducing the cost of ovarian \\
stimulation
\end{tabular}

Abbreviation: IVF, in vitro fertilization. subfertility can account for nearly $40 \%$ of patients seeking fertility treatment. ${ }^{15}$ Without tubal pathology and severe malefactor subfertility, the natural conception rate can be $>50 \%$ if expectant management is adopted. ${ }^{14,16}$ Economic-modeling studies reveal that in younger women with unexplained subfertility, IVF treatment is not cost effective within 3 years of trying to conceive. ${ }^{14,17}$ One randomized controlled trial estimated that for couples who presented with a year's history of unexplained subfertility, a saving of $€ 2,616$ per couple can be achieved if expectant management is adopted for 6 months before fertility treatment is offered..$^{18}$ These results indicate that cost savings can be achieved by careful patient selection, thus avoiding unnecessary interventions and costs when expectant management should be practiced in the first instance.

Intrauterine insemination (IUI) is a well-established treatment and has been offered as a less-expensive alternative to IVF in assisted reproduction. Although there are studies reporting that IUI has no significant advantages over expectant management in unexplained infertility, ${ }^{16,19,20}$ in many parts of the world IUI is still a routine practice. For example, in the United States, the percentages of subfertile patients receiving IUI and IVF treatments are similar. ${ }^{21}$ In the United Kingdom, the most recent guideline on fertility assessment and treatment by National Institute for Health and Clinical Excellence (NICE) does not recommend IUI for couples with unexplained infertility; ${ }^{2}$ this method of treatment is not deemed to be cost effective.

\section{Suggested strategies to reduce the cost of IVF treatment Simplifying investigative methods}

Simple, rather inexpensive baseline investigations, along with an adequate medical history, may be all that is required to reach a diagnosis of a couple's subfertility problem. Avoiding waste such as duplicate and unnecessary investigations may further reduce the average cost of investigations. ${ }^{22,23}$ Studies have demonstrated that the practices involved in investigating subfertility are hugely variable among developed countries. ${ }^{23}$ Even in a single secondary referral center, inconsistencies in basic investigative methods are present; the lack of standard protocols can lead to large differences in the cost of investigations among various practices. ${ }^{22}$

Male-factor subfertility can be identified by semen analysis using a light microscope..$^{24,25}$ This manual method of semen analysis is the recommended standard for assessing sperm. ${ }^{26}$ The reliability of manual semen analysis has not been surpassed by the more expensive computer-aided sperm 
analysis system. ${ }^{26,27}$ Although assessment of pelvic anatomy and ovarian reserve by antral follicle count (AFC) requires an ultrasound scan machine with adequate resolution, advanced digital function or three-dimensional imaging is not necessary and has not been shown to provide any advantage. ${ }^{28}$

One could argue the need to perform tubal patency tests if the decision to offer IVF treatments will not be altered by the results. In the United Kingdom, NICE recommends IVF as the treatment of choice for most cases of subfertility. Cumulatively, IVF is considered the most cost-effective treatment for all subfertility factors apart from anovulation, where ovulation induction is recommended. ${ }^{2}$ Therefore, the value of assessing tubal patency has been debated; there are suggestions that the resources used for these investigations should be channeled to fund IVF instead. However, in specific groups of patients, the knowledge of their tubal status can influence the mode of treatment offered; thus, in many centers, tubal patency tests are routinely conducted, and decisions are made depending on the results. In cases where the tubal status has to be investigated, hysterosalpingography (HSG) or hysterosalpingo-contrast-sonography can be performed. These tubal assessment techniques are comparatively inexpensive and have been recommended as the investigative methods of choice for patients with no other comorbidities. ${ }^{2,10}$ Some other patients may need more-expensive investigative methods to assess tubal status; careful selection of appropriate options can help to avoid unnecessary costs. Laparoscopy is the gold standard for the diagnosis of tubal patency and also has a higher prognostic significance in predicting future fertility; ${ }^{29}$ therapeutic procedures, for example treatments for adhesions, endometriosis, and ovarian drilling, can be performed during laparoscopy. Clinical and cost effectiveness of the above methods for tubal assessment has been evaluated. Compared to laparoscopy as a test for tubal obstruction, HSG provided pooled estimates of sensitivity of 0.65 and specificity of 0.83 , with a cost difference of $€ 753$. $^{2,30,31}$ Hystero-salpingo-contrast sonography has been shown to exhibit good statistical comparability and concordance with HSG. ${ }^{2,32}$

Hysteroscopy may be necessary to investigate and treat submucosal fibroids, polyps, septa, and intrauterine adhesions for patients suspected to have these intrauterine abnormalities. ${ }^{10}$ These procedures are more expensive but are typically performed as day cases to avoid the expenditure of inpatient hospital stay. With the invention of office endoscopic instruments and techniques, some of the procedures can now be performed safely using an ambulatory approach in "one-stop subfertility clinics"; the technique of transvaginal hydrolaparoscopy allows direct visualization of the reproductive organs and the assessment of tubal patency in an outpatient setting. ${ }^{33}$ This method has been clinically validated for accuracy, safety, and patient tolerance. ${ }^{34,35}$ It is commonly performed in the People's Republic of China and some European countries but is not frequently performed in the United Kingdom or United States. ${ }^{36}$ To effectively provide the above services for the investigation of subfertility, careful planning of service delivery utilizing the available resources and the adequate training of staff are essential.

It is widely accepted that ovarian reserve testing is important during the workup for women before undergoing IVF treatment. Ovarian stimulation is a balancing act: the aim is to achieve adequate oocyte yield without causing ovarian hyperstimulation syndrome (OHSS). In this day and age, inducing ovarian stimulation without the predictive value of individual ovarian potential is deemed oversimplistic and dangerous. ${ }^{37}$ Biomarkers of ovarian response have enhanced the practitioners' ability to determine the type, dosage, and duration of the agents used for ovarian stimulation to enhance safety and to improve clinical and cost effectiveness. In one study, the researchers derived a Markov decision model to compare the cost-effectiveness of ovarian reserve testing in IVF. ${ }^{38}$ A computer-simulated cohort of subfertile women aged 20-45 years who are eligible for IVF was generated. The incremental cost-effectiveness ratios per additional live birth was greatly reduced when dose individualization of the follicle-stimulating hormone according to ovarian reserve testing was practiced, in comparison with initiating IVF treatment without any information on ovarian reserve. Although the effectiveness of this method needs to be confirmed in randomized clinical trials, sensitivity analysis revealed that the model was robust.

The two main biomarkers that can reliably predict the oocytes yield from ovarian stimulation are AFC and antiMüllerian hormone (AMH). These two tests have been proven to exhibit strong associations with the size of the primordial follicle pool and follicular recruitment rates. ${ }^{39-41}$ Analyses of AFC against AMH have consistently demonstrated strong correlations in the readings of these two tests. ${ }^{41-43}$ Thus, it is believed that the same $2-6 \mathrm{~mm}$ follicles seen on ultrasound are those producing AMH. ${ }^{41,44}$ In the United Kingdom, NICE recommends that either one of the above two tests or follicle-stimulating hormone (FSH) should be used to predict ovarian response. ${ }^{2} \mathrm{FSH}$ alone is less reliable than $\mathrm{AFC}$ and $\mathrm{AMH}$, as the level fluctuates more widely within a menstrual cycle. To keep the cost of fertility investigations down, the levels of either AMH or AFC alone, but not both, are sufficient for determining the individualized protocols 
for ovarian stimulation for most patients. In the presence of an ultrasound machine with sufficient image resolution and trained personnel, AFC can be measured during pelvic assessment in a subfertility outpatient setting. As mentioned above, sophisticated machines with three-dimensional images or automated counting of antral follicles are usually not required. To ensure the reliability of the results, adequate training is essential for the staff performing the measurement of AFC. Predicting ovarian response by measuring AFC is prone to interobserver variation; ${ }^{41}$ by introducing strict inclusion criteria for AFC measurement, one can reduce the scale of the inconsistency.

$\mathrm{AMH}$, despite its cost, can be measured without the need for any additional clinical skills. Patients can have this test without the need to travel to a tertiary fertility center. This is helpful if the center is providing fertility care for a wide geographical area. As the number of AMH tests increases, the cost to run the samples may decrease over time. However, the result of $\mathrm{AMH}$ is sensitive to the changes in sample handling, storage, and processing; elevated and suppressed levels of serum AMH have been shown to be associated with abnormal batches of calibrators, inappropriate use of linear rather than cubic regression for standard curve interpretation, sample collection in EDTA (ethylenediaminetetraacetic acid) tubes rather than serum tubes, postage of samples before centrifuge, storage at room temperature, and poor operator reproducibility. ${ }^{41,45}$ In addition, there are more than five different assays for AMH measurement from different companies. ${ }^{41}$ Laboratory and clinical personnel need to be knowledgeable in processing a particular assay and in interpreting the results. Due to the lack of international standardization, the external validity of the $\mathrm{AMH}$ results is poor. An AMH result may not be relevant or, in a worst-case scenario, may interpreted incorrectly when a patient moves to a different IVF center for treatment.

Currently, neither of these two methods (AMH and AFC) has been shown to be directly correlated to the clinical pregnancy rate from IVF. ${ }^{2,46}$ Considering the strengths and limitations of these two tests, various IVF practitioners have adopted either one or both tests for predicting ovarian response; their decisions typically depend on their personal preference and the availability of the resources in their centers. In general, it has been perceived that in centers where the AMH facility is not already set up, the most cost-effective method to accurately measure ovarian reserve is AFC. The result of a large-scale multicenter randomized controlled trial to assess the cost effectiveness of AFC to individualize FSH stimulation dosages for IVF treatment will be published in the near future. ${ }^{47}$

\section{Reducing the cost of ovarian stimulation}

The price of medications used for pituitary downregulation and ovarian stimulation is a major contributing factor to the high cost of IVF treatment. The clinical and cost effectiveness of various IVF protocols has been researched. Recently, there has been some renewed interest in natural cycle and mild ovarian stimulation IVF protocols. In natural cycle IVF, no ovarian stimulatory drugs are administered. Ovulation triggering with human chorionic gonadotropin is administered when the follicle matures, reaching the size of 15-20 mm, or when serum estradiol level rises. ${ }^{48}$ A study in 2001 involving 52 women with a total of 181 cycles of treatment revealed that natural cycle IVF has a cumulative probability of pregnancy of $46 \%$ and a live birth rate of $32 \%$ after four cycles of treatment. ${ }^{49}$ With the avoidance of expensive drugs, natural cycle IVF was considered a cost-effective alternative to conventional IVF techniques. ${ }^{49}$ However, a recent metaanalysis revealed that stimulation with clomiphene citrate, with a small increase in cost, has an improved clinical pregnancy rate in comparison with natural cycle IVF. ${ }^{2,50,51}$ The major issue with natural cycle IVF is its high cancellation rates due to premature luteinizing hormone $(\mathrm{LH})$ rise and ovulation; thus overall, the current evidence does not favor natural cycle IVF. ${ }^{10,52}$

In mild ovarian stimulation, a short stimulation period of 2-6 days during the mid-to-late stimulation phase is introduced using FSH, combined with a gonadotropin-releasing hormone $(\mathrm{GnRH})$ antagonist at approximately the same time. ${ }^{48}$ This method allows undisturbed recruitment of a cohort of follicles during the early follicular phase in the IVF treatment cycle, reducing the dose of FSH needed for ovarian stimulation. The treatment also reduces the cancellation rates seen in natural cycle IVF due to premature LH rises and ovulation. ${ }^{53}$ According to the International Society for Mild Approaches in Assisted Reproduction consensus group, using the mild ovarian stimulation protocol, the number of oocytes retrieved usually ranges from two to seven. ${ }^{48}$ Some centers practice modified natural cycle IVF, where a single follicle is allowed to naturally develop and assume dominance. In these cycles, FSH is administered for a period of 2-6 days, starting at different stages depending on the practice of the centers. To suppress LH secretion to prevent premature ovulation, a GnRH antagonist is administered either when the largest follicle reaches a diameter of $14 \mathrm{~mm}$ or after up to 6 days of ovarian stimulation with FSH. When the leading follicle reaches $15-20 \mathrm{~mm}$, ovulation is triggered in the same manner as conventional IVF, and only one oocyte is retrieved for the preparation of a single embryo transfer. ${ }^{48,54}$ 
The technique of in vitro maturation of oocytes can potentially reduce the amount of FSH required for ovarian stimulation. However, the lowered cost of FSH may be offset by increased expenditure in the laboratory. Information on the clinical and cost effectiveness of this technique is needed for the evaluation of its routine application in IVF treatments.

It has been suggested that although mild ovarian stimulation results in a lower oocytes yield, the oocytes retrieved are of a better quality. In mild ovarian stimulation, there is minimal interference with the natural selection of good-quality oocytes; the growing follicles also have less exposure to the potentially negative effects of ovarian stimulatory agents. ${ }^{53}$ In a randomized trial, it was discovered that a significantly higher proportion of euploid embryos formed using the mild stimulation approach compared with conventional stimulation. ${ }^{55}$ When comparing mild stimulation with conventional IVF, recent meta-analyses failed to reveal any differences in cumulative live births, OHSS, or ongoing pregnancy. Due to the heterogeneity of the trials involved, the clinical superiority or noninferiority of one approach over the other could not be concluded. ${ }^{48}$ Per IVF cycle in the mild stimulation approach, there are lower pregnancy rates; due to the fewer number of embryos retrieved, the number available for cryopreservation is usually small. This method also requires excellent laboratory performance, as the margin for error and subpar performance is lower. Few studies have compared the cumulative cost-effectiveness of mild stimulation with conventional controlled ovarian hyperstimulation in IVF. Although in each cycle the amount of drugs administered using the mild stimulation protocol is considerably less, the patients may need to undergo more treatment cycles due to the lower pregnancy rate per cycle of treatment. One recent retrospective study found that the modified natural cycle was less cost effective and had a lower cumulative live birth rate in comparison with conventional IVF. ${ }^{54}$ In summary, when appropriate clinical expertise and laboratory resources are available, there may be a place for mild ovarian stimulation IVF for selected patients.

GnRH agonists and GnRH antagonists are the two most commonly used drugs for pituitary downregulation in IVF treatment. GnRH antagonists generally cost more than GnRH agonists. However, in GnRH antagonist protocols, this medication is used for a shorter portion of the treatment cycle to achieve the same downregulatory effect. Thus, the price difference between these two protocols may not be large. A study in the United States indicated that on average, the cost of the GnRH agonist protocol is as much as USD $\$ 400$ lower than that of the GnRH antagonist protocol. ${ }^{56}$
Thus, if the costs from this study can be applied universally, a GnRH agonist is generally the more cost-effective option in a limited resource setting. The risk of OHSS is higher in the GnRH agonist protocol; ;,57 in high-risk patients, GnRH antagonists are preferable.

Although urinary and recombinant gonadotropins are the most researched and preferred regimens for ovarian stimulation, clomiphene citrate (clomiphene) was the first preparation used for ovarian stimulation in IVF. ${ }^{53,58}$ Although Edwards and Steptoe tried and then largely abandoned both this medication and tamoxifen, ${ }^{59,60}$ others have continued to demonstrate that clomiphene can be a cost-effective way to achieve full-term IVF pregnancies. ${ }^{60}$ The advantages of clomiphene include oral administration, low price, and widespread availability in many parts of the world. ${ }^{53}$ Clomiphene can be used as a sole stimulatory medication in mild ovarian stimulation IVF. As mentioned above, the clinical pregnancy rate in IVF stimulation by clomiphene is superior to natural cycle IVF. The major disadvantage of clomiphene is its antiestrogenic effect on the endometrium, which may adversely affect the receptivity for embryo implantation. ${ }^{61,62}$ Clomiphene may be combined with exogenous gonadotropins, with or without GnRH antagonists, to prevent a spontaneous LH surge. This practice reduces the total number of ampoules of gonadotropins needed, thus lowering the cost of treatment. ${ }^{63}$ Several studies have reported that this option does not affect the pregnancy rates of IVF treatment; ${ }^{53,64-66}$ however, one study indicated that combining clomiphene and gonadotropin results in a higher cancellation rate and lowers the ongoing pregnancy rate. ${ }^{63}$ Although more studies are needed to confirm the clinical and cost effectiveness of using clomiphene with or without gonadotropins and $\mathrm{GnRH}$ antagonists, overall, these are attractive methods for reducing the cost of IVF treatment.

Aromatase inhibitors, eg, letrozole, can be used for the same purpose as clomiphene. These drugs inhibit the conversion of androgens to estrogens in granulosa cells in developing ovarian follicles, thus preventing the rise in the level of estrogens. ${ }^{53,67}$ This results in a reduction in estrogen feedback and subsequently in an increase in endogenous gonadotropin secretion. Aromatase inhibitors have an additional advantage of not causing depletion of estrogen receptors. ${ }^{53,68}$ There are few reports of trials involving aromatase inhibitors, with most of them combining letrozole with gonadotropins for ovarian stimulation. One uncontrolled study favored aromatase inhibitors as effective and inexpensive treatment alternatives; the study reported an ongoing pregnancy rate of $27 \%$ following treatment using 
letrozole and a reduced amount of human menopausal gonadotropin. ${ }^{69}$ Another study reported that the addition of letrozole to recombinant FSH increased the oocytes yield and resulted in a tendency towards higher clinical pregnancy rates; however, in this study, the dose of FSH was the same between the groups compared, and thus no cost saving was made. ${ }^{70}$ In patients with poor prognosis, the addition of aromatase inhibitors does not improve outcomes. ${ }^{53,71}$ At present, more information on cumulative clinical effectiveness and cost effectiveness is needed to confirm the efficacy of this IVF treatment regimen. In summary, it seems appropriate to use aromatase inhibitors in a limited resource setting if they reduce the cost of the treatment.

\section{Simplifying procedures and equipment in the laboratory}

Over the years, the laboratorial procedures in IVF treatment have become increasingly complex. Additional interventions have been supplemented in IVF laboratories; not all of them are proven to be beneficial. Intracytoplasmic sperm injection (ICSI) was invented in 1991; this supplementary step in IVF has been shown to be beneficial for a subgroup of patients, namely those with male-factor subfertility. With the current evidence, this intervention is recommended in the United Kingdom for couples with severe deficits in semen quality, those with obstructive and nonobstructive azoospermia, and those in whom a previous IVF treatment cycle has resulted in failed or very poor fertilization. ${ }^{2}$ Preimplantation genetic screening had a promising start but subsequently was reported to reduce the live birth rate. ${ }^{72}$ Another new invention, the embryoscope, has lately been very sought after by IVF laboratories and patients; however, the long-term data on the clinical and cost effectiveness of this system is lacking. The robotic ICSI method is often mentioned; this technology may become commercially available in the near future. ${ }^{73}$ The embryo selection techniques, such as metabolomic profiling of culture medium, failed to exhibit any benefit in a randomized controlled trial. ${ }^{74,75}$ Spermatic assessment and selection has attracted tremendous attention in recent years. It has been suggested that sperm DNA fragmentation greater than $25.5 \%$ is associated with a higher probability of unsuccessful IVF treatment. ${ }^{76}$ However, sperm selection techniques, such as intracytoplasmic morphologically selected sperm injection, that select spermatozoa at high magnification have not been shown to improve outcomes in studies, including a multicentric randomized controlled trial. ${ }^{76,77}$ The effectiveness of sperm selection for ICSI by prior hyaluronic acid binding is currently being investigated in the large multicenter randomized HABSelect trial in the United Kingdom.

In an environment with abundant resources, it is not unreasonable to explore new horizons in IVF treatment to make an effort to improve outcomes. However, one has to acknowledge that the cost of IVF treatment may be substantially increased with any additional laboratory intervention. The cost to set up an IVF laboratory is hardly economical, at usually between $€ 1.5-€ 3.0$ million. ${ }^{78}$ Researchers in the past have indeed tried to reduce the expenditure of laboratory procedures in IVF. There have been reports of intravaginal fertilization and incubation of oocytes and sperms. ${ }^{10,79}$ This technique has not been widely accepted and adopted. Recently, there was a breakthrough by researchers in Belgium. ${ }^{78}$ The project, supported by The Walking Egg Foundation, resulted in the possibility of setting up a low-cost IVF laboratory for less than $€ 300,000$. The team developed a closed culture system using an inexpensive, disposable $10 \mathrm{~mL}$ plain glass vacutainer. With the single-tube method, the researchers could adequately visualize oocytes, pronuclear eggs, and preimplantation embryos to the hatched blastocyst stage. This setup enables stage and performance assessments of sufficient detail to select single embryos for transfer. To generate a stable medium $\mathrm{pH}$ and atmospheric conditions like tissue culture incubators, pure $\mathrm{CO}_{2}$ was produced by the combination of sodium bicarbonate, citric acid, and water. With this simplified culture system, it was reported that eight of 23 embryos implanted, one miscarried at 8 weeks, and seven healthy infants were born; the total laboratory expenses for this system are only $10 \%-15 \%$ of those used by most IVF laboratories. ${ }^{80}$ The preliminary result indicates that good live birth rates and tremendous cost savings can be achieved by adapting to this laboratory technique. The system has the disadvantage that it is currently not suitable for ICSI. Nevertheless, bilateral tubal occlusion is prevalent in some developing countries. Without the presence of male-factor subfertility, conventional IVF without ICSI intervention is the recommended treatment for this group of patients. Therefore, this simplified culture system is particularly effective in this setting. Overall, this exciting discovery demonstrates that IVF laboratories can be run on a shoestring budget, thus improving the accessibility of the treatment to a wider population, including those in developing countries.

\section{Minimizing the complications of IVF}

In a competitive world, IVF centers are increasingly focused on staying at the top of the league tables of success rates; patients often choose the centers with the best success rates without paying much attention to other factors like the 
rates of the two most common complications, which are OHSS and multiple pregnancies..$^{81,82}$

Severe and critical OHSS not only endangers the life of women undergoing IVF but also imposes tremendous pressure on the healthcare resources. These patients often need the input from a multidisciplinary team in an intensive care environment. Bob Edwards has warned that high-order ovarian stimulation could be injurious to women's health and called for a reassessment of the reliance on complex, costly treatment protocols that result in large numbers of oocytes:

[...] endocrinological approaches, preferably reinforcing the natural cycle and acceptable for successive cycles of mild stimulation, are needed today. At most, modest forms of ovarian stimulation aimed at stimulating the growth of a limited number of Graafian follicles should suffice. ${ }^{60,83,84}$

IVF has single-handedly increased the rate of multiple pregnancies throughout the world. Multiple pregnancies are associated with high rates of pregnancy-related and neonatal complications. Approximately 50\% of twin pregnancies are associated with preterm delivery. Two-thirds of twins are small for gestational age. ${ }^{10}$ Approximately $7.4 \%, 21.6 \%$, and $50 \%$ of twin, triplet, and quadruplet pregnancies, respectively, produce at least one handicapped child. ${ }^{85}$ In comparison to singletons, twins and triplets are 6 times and 10 times more likely, respectively, to have cerebral palsy. ${ }^{10,86}$ In addition, the risk of neonatal complications of IVF twins is greater than in spontaneously conceived dizygotic twins.$^{87}$ In the United Kingdom, it has been shown that $56 \%$ of the direct cost of IVF pregnancies is associated with multiple pregnancies after IVF. The total direct maternal and infant costs per IVF singleton family is only $£ 3,313$, in comparison with $£ 9,122$ per IVF twin family and $£ 32,354$ per IVF triplet family; ${ }^{88}$ the cost related to triplet pregnancies is almost 10 times the cost for singleton pregnancies.

The practice of elective single embryo transfer has been shown to drastically reduce the incidence of IVF multiple pregnancies and the associated complications..$^{10,89}$ As mentioned above, some health boards impose the policy of elective single embryo transfer as one of the criteria for accessing the funding for treatment. In some countries, the authorities monitor the rates of multiple pregnancies at IVF centers. In Australia, the costs saved by reducing multiple pregnancies were calculated in one study. The reduction of fertility treatment-related multiple birth rate from $18.8 \%$ to $8.6 \%$ has resulted in the savings of AUD $\$ 47.6$ million, which is equivalent to a $55 \%$ increase in use of the service. ${ }^{90}$

\section{The future of IVF for patients with subfertility}

In conclusion, the most significant breakthrough in reducing the cost of IVF in recent years is the invention of a simplified culture system. It is exciting that laboratory costs can be reduced by up to $90 \%$. This innovation demonstrates that affordable IVF, especially in developing countries with very basic resources, is within reach in the not-too-distant future. Low-cost medication for ovarian stimulation can be chosen to further lower the cost of IVF. The Arusha Project, an offspring of The Walking Egg, has made major contributions in increasing the public awareness of the need for subfertility services in developing countries and also in discovering the mechanism for the provision of IVF treatment in low-resource settings. It is known that the availability of equipment itself is not sufficient for effective IVF service delivery. The provision of training and education in the practicalities and risks of IVF to service providers are important factors in ensuring safe and effective utilization of the facilities. Well-trained, informed clinicians and IVF laboratory scientists are required for the establishment and maintenance of a high-quality service. There is also a need to increase the public awareness of the limits and risks of IVF; well-supported campaigns, such as that found at http://www. oneatatime.org.uk, have the aim of attracting attention from the public and improving the understanding of complications from IVF treatment.

In summary, the current trend for the development of low-cost IVF is very encouraging. Recent discoveries should prompt international organizations to enhance their support in bringing this effective service to the people in need of IVF treatment in areas with limited resources. With continued support for further research and service development, millions of people troubled by subfertility can be offered help; those that are financially disadvantaged will not have to accept the fate and bear the sorrow of childlessness.

\section{Disclosure}

The authors report no conflicts of interest in this work.

\section{References}

1. Maheshwari A, Scotland G, Bell J, McTavish A, Hamilton M, Bhattacharya S. The direct health services costs of providing assisted reproduction services in overweight or obese women: a retrospective cross-sectional analysis. Hum Reprod. 2009;24(3):633-639.

2. National Institute for Health and Care Excellence. Fertility: assessment and treatment for people with fertility problems [webpage on the Internet]. London, UK: National Institute for Health and Care Excellence; 2013. Available from: http://guidance.nice.org.uk/CG156/Guidance/pdf/ English. Accessed March 11, 2014. 
3. Aleyamma TK, Kamath MS, Muthukumar K, Mangalaraj AM, George K. Affordable ART: a different perspective. Hum Reprod. 2011;26(12):3312-3318.

4. Chambers GM, Hoang VP, Illingworth PJ. Socioeconomic disparities in access to ART treatment and the differential impact of a policy that increased consumer costs. Hum Reprod. 2013;28(11):3111-3117.

5. Smith JF, Eisenberg ML, Glidden D, et al. Socioeconomic disparities in the use and success of fertility treatments: analysis of data from a prospective cohort in the United States. Fertil Steril. 2011;96(1):95-101.

6. Rauprich O, Berns E, Vollmann J. Who should pay for assisted reproductive techniques? Answers from patients, professionals and the general public in Germany. Hum Reprod. 2010;25(5):1225-1233.

7. Murage A, Muteshi MC, Githae F. Assisted reproduction services provision in a developing country: time to act? Fertil Steril. 2011;96(4):966-968.

8. Makuch MY, Petta CA, Osis MJ, Bahamondes L. Low priority level for infertility services within the public health sector: a Brazilian case study. Hum Reprod. 2010;25(2):430-435.

9. Makuch MY, Simônia de Padua K, Petta CA, Duarte Osis MJ, Bahamondes L. Inequitable access to assisted reproductive technology for the low-income Brazilian population: a qualitative study. Hum Reprod. 2011;26(8):2054-2060.

10. Ombelet W, Cooke I, Dyer S, Serour G, Devroey P. Infertility and the provision of infertility medical services in developing countries. Hum Reprod Update. 2008;14(6):605-621.

11. Dyer SJ, Abrahams N, Mokoena NE, Lombard CJ, van der Spuy ZM. Psychological distress among women suffering from couple infertility in South Africa: a quantitative assessment. Hum Reprod. 2005;20(7):1938-1943.

12. Ombelet W. Is global access to infertility care realistic? The Walking Egg Project. Reprod Biomed Online. 2014;28(3):267-272.

13. The walking egg: global access to infertility care [homepage on the Internet]. The Walking Egg; 2014. Available from: http://thewalkingegg. com. Accessed July 27, 2014.

14. Kamphuis EI, Bhattacharya S, van der Veen F, Mol BW, Templeton A; Evidence Based IVF Group. Are we overusing IVF? BMJ. 2014;348:g252.

15. Farquhar CM, van den Boogaard NM, Riddell C, Macdonald A, Chan E, Mol BW. Accessing fertility treatment in New Zealand: a comparison of the clinical priority access criteria with a prediction model for couples with unexplained subfertility. Hum Reprod. 2011;26(11):3037-3044.

16. Steures P, van der Steeg JW, Hompes PG, et al; Collaborative Effort on the Clinical Evaluation in Reproductive Medicine. Intrauterine insemination with controlled ovarian hyperstimulation versus expectant management for couples with unexplained subfertility and an intermediate prognosis: a randomised clinical trial. Lancet. 2006;368(9531): 216-221.

17. Mol BW, Bonsel GJ, Collins JA, Wiegerinck MA, van der Veen F, Bossuyt PM. Cost-effectiveness of in vitro fertilization and embryo transfer. Fertil Steril. 2000;73(4):748-754.

18. Custers IM, van Rumste MM, van der Steeg JW, et al; CECERM. Longterm outcome in couples with unexplained subfertility and an intermediate prognosis initially randomized between expectant management and immediate treatment. Hum Reprod. 2012;27(2):444-450.

19. Bhattacharya S, Harrild K, Mollison J, et al. Clomifene citrate or unstimulated intrauterine insemination compared with expectant management for unexplained infertility: pragmatic randomised controlled trial. BMJ. 2008;337:a716.

20. Tummon IS, Asher LJ, Martin JS, Tulandi T. Randomized controlled trial of superovulation and insemination for infertility associated with minimal or mild endometriosis. Fertil Steril. 1997;68(1):8-12.

21. Farland LV, Missmer SA, Rich-Edwards J, Chavarro JE, Barbieri RL, Grodstein F. Use of fertility treatment modalities in a large United States cohort of professional women. Fertil Steril. 2014;101(6):1705-1710.

22. Ojha K, Philips Z, Darne FJ. Diagnosing infertility in a district general hospital: a case-note and cost analysis. Hum Fertil (Camb). 2003;6(4):169-173.
23. Helmerhorst FM, Oei SG, Bloemenkamp KW, Keirse MJ. Consistency and variation in fertility investigations in Europe. Hum Reprod. 1995;10(8):2027-2030.

24. Menkveld R, Wong WY, Lombard CJ, et al. Semen parameters, including WHO and strict criteria morphology, in a fertile and subfertile population: an effort towards standardization of in-vivo thresholds. Hum Reprod. 2001;16(6):1165-1171.

25. Gunalp S, Onculoglu C, Gurgan T, Kruger TF, Lombard CJ. A study of semen parameters with emphasis on sperm morphology in a fertile population: an attempt to develop clinical thresholds. Hum Reprod. 2001;16(1):110-114.

26. World Health Organization. WHO Laboratory Manual for the Examination and Processing of Human Semen. 5th ed. Geneva, Switzerland: World Health Organization; 2010.

27. Tomlinson MJ, Pooley K, Simpson T, et al. Validation of a novel computer-assisted sperm analysis (CASA) system using multitargettracking algorithms. Fertil Steril. 2010;93(6):1911-1920.

28. Jayaprakasan K, Hilwah N, Kendall NR, et al. Does 3D ultrasound offer any advantage in the pretreatment assessment of ovarian reserve and prediction of outcome after assisted reproduction treatment? Hum Reprod. 2007;22(7):1932-1941.

29. Mol BW, Collins JA, Burrows EA, van der Veen F, Bossuyt PM. Comparison of hysterosalpingography and laparoscopy in predicting fertility outcome. Hum Reprod. 1999;14(5):1237-1242.

30. Verhoeve HR, Moolenaar LM, Hompes P, van der Veen F, Mol BW. Cost-effectiveness of tubal patency tests. BJOG. 2013;120(5):583-593.

31. Swart P, Mol BW, van der Veen F, van Beurden M, Redekop WK, Bossuyt PM. The accuracy of hysterosalpingography in the diagnosis of tubal pathology: a meta-analysis. Fertil Steril. 1995;64(3):486-491.

32. Dijkman AB, Mol BW, van der Veen F, Bossuyt PM, Hogerzeil HV. Can hysterosalpingocontrast-sonography replace hysterosalpingography in the assessment of tubal subfertility? Eur J Radiol. 2000; 35(1):44-48.

33. Brosens I, Campo R, Puttemans P, Gordts S. One-stop endoscopy-based infertility clinic. Curr Opin Obstet Gynecol. 2002;14(4):397-400.

34. Cicinelli E, Matteo M, Causio F, Schonauer LM, Pinto V, Galantino P. Tolerability of the mini-pan-endoscopic approach (transvaginal hydrolaparoscopy and minihysteroscopy) versus hysterosalpingography in an outpatient infertility investigation. Fertil Steril. 2001;76(5):1048-1051.

35. Watrelot A, Nisolle M, Chelli H, Hocke C, Rongières C, Racinet C; International Group for Fertiloscopy Evaluation. Is laparoscopy still the gold standard in infertility assessment? A comparison of fertiloscopy versus laparoscopy in infertility. Results of an international multicentre prospective trial: the 'FLY' (Fertiloscopy-LaparoscopY) study. Hum Reprod. 2003;18(4):834-839.

36. Ezedinma NA, Phelps JY. Transvaginal hydrolaparoscopy. JSLS. 2012;16(3):461-465.

37. Nardo LG, Fleming R, Howles CM, et al. Conventional ovarian stimulation no longer exists: welcome to the age of individualized ovarian stimulation. Reprod Biomed Online. 2011;23(2):141-148.

38. Moolenaar LM, Broekmans FJ, van Disseldorp J, et al. Cost effectiveness of ovarian reserve testing in in vitro fertilization: a Markov decision-analytic model. Fertil Steril. 2011;96(4):889-894.

39. Nelson SM, Anderson RA, Broekmans FJ, Raine-Fenning N, Fleming R, La Marca A. Anti-Müllerian hormone: clairvoyance or crystal clear? Hum Reprod. 2012;27(3):631-636.

40. Kelsey TW, Anderson RA, Wright P, Nelson SM, Wallace WH. Data-driven assessment of the human ovarian reserve. Mol Hum Reprod. 2012;18(2):79-87.

41. Nelson SM. Biomarkers of ovarian response: current and future applications. Fertil Steril. 2013;99(4):963-969.

42. Nardo LG, Christodoulou D, Gould D, Roberts SA, Fitzgerald CT, Laing I. Anti-Müllerian hormone levels and antral follicle count in women enrolled in in vitro fertilization cycles: relationship to lifestyle factors, chronological age and reproductive history. Gynecol Endocrinol. 2007;23(8):486-493. 
43. van Disseldorp J, Lambalk CB, Kwee J, et al. Comparison of inter- and intra-cycle variability of anti-Mullerian hormone and antral follicle counts. Hum Reprod. 2010;25(1):221-227.

44. Weenen C, Laven JS, Von Bergh AR, et al. Anti-Müllerian hormone expression pattern in the human ovary: potential implications for initial and cyclic follicle recruitment. Mol Hum Reprod. 2004;10(2): 77-83.

45. Fleming R, Nelson SM. Reproducibility of AMH. Hum Reprod. 2012;27(12):3639-3641; author reply 3641

46. Vural B, Cakiroglu Y, Vural F, Filiz S. Hormonal and functional biomarkers in ovarian response. Arch Gynecol Obstet. 2014;289(6): 1355-1361.

47. van Tilborg TC, Eijkemans MJ, Laven JS, et al. The OPTIMIST study: optimisation of cost effectiveness through individualised FSH stimulation dosages for IVF treatment. A randomised controlled trial. BMC Womens Health. 2012;12:29.

48. Allersma T, Farquhar C, Cantineau AE. Natural cycle in vitro fertilisation (IVF) for subfertile couples. Cochrane Database Syst Rev. 2013;8:CD010550.

49. Nargund G, Waterstone J, Bland J, Philips Z, Parsons J, Campbell S. Cumulative conception and live birth rates in natural (unstimulated) IVF cycles. Hum Reprod. 2001;16(2):259-262.

50. Ingerslev HJ, Højgaard A, Hindkjaer J, Kesmodel U. A randomized study comparing IVF in the unstimulated cycle with IVF following clomiphene citrate. Hum Reprod. 2001;16(4):696-702.

51. MacDougall MJ, Tan SL, Hall V, Balen A, Mason BA, Jacobs HS. Comparison of natural with clomiphene citrate-stimulated cycles in in vitro fertilization: a prospective, randomized trial. Fertil Steril. 1994;61(6):1052-1057.

52. Pelinck MJ, Hoek A, Simons AH, Heineman MJ. Efficacy of natural cycle IVF: a review of the literature. Hum Reprod Update. 2002;8(2):129-139.

53. Verberg MF, Macklon NS, Nargund G, et al. Mild ovarian stimulation for IVF. Hum Reprod Update. 2009;15(1):13-29.

54. Groen H, Tonch N, Simons AH, van der Veen F, Hoek A, Land JA. Modified natural cycle versus controlled ovarian hyperstimulation IVF: a cost-effectiveness evaluation of three simulated treatment scenarios. Hum Reprod. 2013;28(12):3236-3246.

55. Baart EB, Martini E, Eijkemans MJ, et al. Milder ovarian stimulation for in-vitro fertilization reduces aneuploidy in the human preimplantation embryo: a randomized controlled trial. Hum Reprod. 2007;22(4):980-988.

56. Sills ES, Collins GS, Salem SA, Jones CA, Peck AC, Salem RD. Balancing selected medication costs with total number of daily injections: a preference analysis of $\mathrm{GnRH}$-agonist and antagonist protocols by IVF patients. Reprod Biol Endocrinol. 2012;10:67.

57. Coan PM, Fowden AL, Constancia M, Ferguson-Smith AC, Burton GJ, Sibley CP. Disproportional effects of Igf2 knockout on placental morphology and diffusional exchange characteristics in the mouse. J Physiol. 2008;586(Pt 20):5023-5032.

58. Trounson AO, Leeton JF, Wood C, Webb J, Wood J. Pregnancies in humans by fertilization in vitro and embryo transfer in the controlled ovulatory cycle. Science. 1981;212(4495):681-682.

59. Edwards RG, Steptoe PC, Purdy JM. Establishing full-term human pregnancies using cleaving embryos grown in vitro. $\mathrm{Br} \mathrm{J}$ Obstet Gynaecol. 1980;87(9):737-756.

60. Hillier SG. IVF endocrinology: the Edwards era. Mol Hum Reprod. 2013;19(12):799-808.

61. Amita M, Takahashi T, Tsutsumi S, et al. Molecular mechanism of the inhibition of estradiol-induced endometrial epithelial cell proliferation by clomiphene citrate. Endocrinology. 2010;151(1):394-405.

62. Siristatidis C, Trivella M, Chrelias C, Sioulas VD, Vrachnis N, Kassanos D. A short narrative review of the feasibility of adopting mild ovarian stimulation for IVF as the current standard of care. Arch Gynecol Obstet. 2012;286(2):505-510.

63. Dhont M, Onghena A, Coetsier T, De Sutter P. Prospective randomized study of clomiphene citrate and gonadotrophins versus goserelin and gonadotrophins for follicular stimulation in assisted reproduction. Hum Reprod. 1995;10(4):791-796.
64. Lin YH, Hwang JL, Seow KM, Huang LW, Hsieh BC, Tzeng CR. Comparison of outcome of clomiphene citrate/human menopausal gonadotropin/cetrorelix protocol and buserelin long protocol - a randomized study. Gynecol Endocrinol. 2006;22(6):297-302.

65. Weigert M, Krischker U, Pöhl M, Poschalko G, Kindermann C, Feichtinger W. Comparison of stimulation with clomiphene citrate in combination with recombinant follicle-stimulating hormone and recombinant luteinizing hormone to stimulation with a gonadotropinreleasing hormone agonist protocol: a prospective, randomized study. Fertil Steril. 2002;78(1):34-39.

66. Gibreel A, Maheshwari A, Bhattacharya S. Clomiphene citrate in combination with gonadotropins for controlled ovarian stimulation in women undergoing in vitro fertilization. Cochrane Database Syst Rev. 2012;11:CD008528.

67. Garcia-Velasco JA, Moreno L, Pacheco A, et al. The aromatase inhibitor letrozole increases the concentration of intraovarian androgens and improves in vitro fertilization outcome in low responder patients: a pilot study. Fertil Steril. 2005;84(1):82-87.

68. Mitwally MF, Casper RF. Aromatase inhibition reduces gonadotrophin dose required for controlled ovarian stimulation in women with unexplained infertility. Hum Reprod. 2003;18(8):1588-1597.

69. Grabia A, Papier S, Pesce R, Mlayes L, Kopelman S, Sueldo C. Preliminary experience with a low-cost stimulation protocol that includes letrozole and human menopausal gonadotropins in normal responders for assisted reproductive technologies. Fertil Steril. 2006;86(4):1026-1028.

70. Verpoest WM, Kolibianakis E, Papanikolaou E, Smitz J, Van Steirteghem A, Devroey P. Aromatase inhibitors in ovarian stimulation for IVF/ICSI: a pilot study. Reprod Biomed Online. 2006;13(2):166-172.

71. Goswami SK, Das T, Chattopadhyay R, et al. A randomized single-blind controlled trial of letrozole as a low-cost IVF protocol in women with poor ovarian response: a preliminary report. Hum Reprod. 2004;19(9):2031-2035.

72. Mastenbroek S, Twisk M, van der Veen F, Repping S. Preimplantation genetic screening: a systematic review and meta-analysis of RCTs. Hum Reprod Update. 2011;17(4):454-466.

73. Lu Z, Zhang X, Leung C, Esfandiari N, Casper RF, Sun Y. Robotic ICSI (intracytoplasmic sperm injection). IEEE Trans Biomed Eng. 2011;58(7):2102-2108.

74. Montag M, Toth B, Strowitzki T. New approaches to embryo selection. Reprod Biomed Online. 2013;27(5):539-546.

75. Hardarson T, Ahlström A, Rogberg L, et al. Non-invasive metabolomic profiling of Day 2 and 5 embryo culture medium: a prospective randomized trial. Hum Reprod. 2012;27(1):89-96.

76. López G, Lafuente R, Checa MA, Carreras R, Brassesco M. Diagnostic value of sperm DNA fragmentation and sperm high-magnification for predicting outcome of assisted reproduction treatment. Asian J Androl. 2013;15(6):790-794.

77. Leandri RD, Gachet A, Pfeffer J, et al. Is intracytoplasmic morphologically selected sperm injection (IMSI) beneficial in the first ART cycle? a multicentric randomized controlled trial. Andrology. 2013;1(5): 692-697.

78. Klerckx E. IVF for 200 euro per cycle: first real-life proof of principle that IVF is feasible and effective for developing countries [press release]. London, UK: European Society of Human Reproduction and Embryology; July 8, 2013. Available from: http://www.eshre. eu/Londen2013/Media/Releases/Elke-Klerckx.aspx. Accessed July 4, 2014.

79. Taymor ML, Ranoux CJ, Gross GL. Natural oocyte retrieval with intravaginal fertilization: a simplified approach to in vitro fertilization. Obstet Gynecol. 1992;80(5):888-891.

80. Van Blerkom J, Ombelet W, Klerkx E, et al. First births with a simplified culture system for clinical IVF and embryo transfer. Reprod Biomed Online. 2014;28(3):310-320.

81. Alper MM, Brinsden PR, Fischer R, Wikland M. Is your IVF programme good? Hum Reprod. 2002;17(1):8-10. 
82. Lass A, Brinsden P. How do patients choose private in vitro fertilization treatment? A customer survey in a tertiary fertility center in the United Kingdom. Fertil Steril. 2001;75(5):893-897.

83. Edwards RG, Lobo R, Bouchard P. Time to revolutionize ovarian stimulation. Hum Reprod. 1996;11(5):917-919.

84. Edwards RG. IVF, IVM, natural cycle IVF, minimal stimulation IVF time for a rethink. Reprod Biomed Online. 2007;15(1):106-119.

85. Yokoyama Y, Shimizu T, Hayakawa K. Incidence of handicaps in multiple births and associated factors. Acta Genet Med Gemellol (Roma). 1995;44(2):81-91.

86. Pharoah PO, Cooke T. Cerebral palsy and multiple births. Arch Dis Child Fetal Neonatal Ed. 1996;75(3):F174-F177.

87. Källén B, Finnström O, Lindam A, Nilsson E, Nygren KG, Olausson PO. Selected neonatal outcomes in dizygotic twins after IVF versus non-IVF pregnancies. BJOG. 2010;117(6):676-682.

88. Ledger WL, Anumba D, Marlow N, Thomas CM, Wilson EC; Cost of Multiple Births Study Group (COMBS Group). The costs to the NHS of multiple births after IVF treatment in the UK. BJOG. 2006;113(1):21-25.
89. McLernon DJ, Harrild K, Bergh C, et al. Clinical effectiveness of elective single versus double embryo transfer: meta-analysis of individual patient data from randomised trials. BMJ. 2010;341: c6945.

90. Chambers GM, Illingworth PJ, Sullivan EA. Assisted reproductive technology: public funding and the voluntary shift to single embryo transfer in Australia. Med J Aust. 2011;195(10):594-598.

91. Chambers GM, Sullivan EA, Ishihara O, Chapman MG, Adamson GD. The economic impact of assisted reproductive technology: a review of selected developed countries. Fertil Steril. 2009;91(6):2281-2294.

92. Widge A. Seeking conception: experiences of urban Indian women with in vitro fertilisation. Patient Educ Couns. 2005;59(3):226-233.

93. Huyser C, Boyd L. ART in South Africa: The price to pay. Facts Views Vis Obgyn. 2013;5(2):91-99.
International Journal of Women's Health

\section{Publish your work in this journal}

The International Journal of Women's Health is an international, peerreviewed open-access journal publishing original research, reports, editorials, reviews and commentaries on all aspects of women's healthcare including gynecology, obstetrics, and breast cancer. The manuscript management system is completely online and includes

\section{Dovepress}

a very quick and fair peer-review system, which is all easy to use. Visit http://www.dovepress.com/testimonials.php to read real quotes from published authors.

\footnotetext{
Submit your manuscript here: http://www.dovepress.com/international-journal-of-womens-health-journal
} 